\title{
STABLE-RANGE APPROACH TO THE EQUATION OF NONSTATIONARY TRANSONIC GAS FLOWS
}

\author{
BY \\ XIAOPING XU \\ Institute of Mathematics, Academy of Mathematics and System Sciences, Chinese Academy of \\ Sciences, Beijing 100080, People's Republic of China
}

\begin{abstract}
Using a certain finite-dimensional stable range of the nonlinear terms, we obtain large families of exact solutions parameterized by functions for the equation of nonstationary transonic gas flows discovered by Lin, Reissner and Tsien and its threedimensional generalization.
\end{abstract}

1. Introduction. Lin, Reissner and Tsien [LRT] found the equation

$$
2 u_{t x}+u_{x} u_{x x}-u_{y y}=0
$$

for two-dimensional nonsteady motion of a slender body in a compressible fluid, which was later called the "equation of nonstationary transonic gas flows" (cf. [M1]). Mamontov obtained the Lie point symmetries of the above equation in [M1] and solved the problem of existence of analytic solutions in [M2]. The three-dimensional generalization

$$
2 u_{t x}+u_{x} u_{x x}-u_{y y}-u_{z z}=0
$$

was studied by Kucharczyk [Kp1] and by Sukhinin [Sv]. Indeed, the Lie point symmetries of the equation (1.2) were found in their works. Sevost'janov [Sg] found explicit solutions of the equation (1.1), describing nonstationary transonic flows in plane nozzles.

In this paper, we present a new approach based on the fact that the nonlinear terms keep some finite-dimensional polynomial space in $x$ stable. We obtain a family of solutions of the equation (1.1) blowing up on a moving line $y=f(t)$, which reflect partial phenomena of gust, and a family of smooth solutions parameterized by six smooth functions of $t$. Moreover, we find a family of solutions of the equation (1.2) blowing up on a rotating and translating plane $\cos \alpha(t) y+\sin \alpha(t) z=f(t)$, which reflect partial phenomena of turbulence, and a family of solutions polynomial in $x$ parameterized by time-dependent harmonic functions in $y$ and $z$, whose special cases are smooth solutions. In particular, we find all the solutions polynomial in $x$ and $y$ for the equation (1.1) and all the solutions polynomial in $x, y$ and $z$ for the equation (1.2). Since our solutions

Received October 2, 2006.

2000 Mathematics Subject Classification. Primary 35C05, 35Q35; Secondary 35C10, 35C15.

Research for this article was supported by China NSF 10431040. 
contain parameter functions, it can be used to solve certain boundary-value problems for these equations.

The Lie group method is one of the most important ways of solving differential equations. However, the method only enables one to obtain certain special solutions. It is desirable to find more effective ways of solving differential equations. Indeed, we do find one way of solving the above nonlinear partial differential equations.

On Mamontov's list of the Lie point symmetries of the equation (1.1) (e.g., cf. page 296 in [I]), the most sophisticated ones are those with respect to the following vector fields:

$$
\begin{aligned}
& X_{1}=3 \alpha(t) \partial_{t}+\left(\alpha^{\prime}(t) x+\alpha^{\prime \prime}(t) y^{2}\right) \partial_{x}+2 \alpha^{\prime}(t) y \partial_{y} \\
& +\left[-\alpha^{\prime}(t) u+\alpha^{\prime \prime}(t) x^{2}+2 \alpha^{\prime \prime \prime}(t) x y^{2}+\frac{1}{3} \alpha^{(4)}(t) y^{4}\right] \partial_{u}, \\
& X_{2}=\beta^{\prime}(t) y \partial_{x}+\beta(t) \partial_{y}+\left[2{\beta^{\prime \prime}}^{\prime}(t) x y+\frac{2}{3}{\beta^{\prime \prime}}^{\prime \prime}(t) y^{3}\right] \partial_{u} \\
& X_{3}=\gamma(t) \partial_{x}+\left[2 \gamma^{\prime}(t) x+2{\gamma^{\prime \prime}}^{\prime}(t) y^{3}\right] \partial_{u}
\end{aligned}
$$

where $\alpha, \beta$ and $\gamma$ are arbitrary functions of $t$. Among the known Lie point symmetries of (1.2) (e.g., cf. page 298 in [I]), the most interesting ones are those with respect to the following vector fields:

$$
\begin{gathered}
X_{4}=\frac{5}{2} t^{2} \partial_{t}+\left(t x+\frac{3}{2}\left(y^{2}+z^{2}\right)\right) \partial_{x}+3 t y \partial_{y}+3 t z \partial_{z}+\left(x^{2}-3 t u\right) \partial_{u} \\
X_{5}=g^{\prime}(t) y \partial_{x}+g(t) \partial_{y}+\left(2 g^{\prime \prime}(t) x y+g^{\prime \prime \prime}(t)\left(\frac{y^{3}}{3}+y z^{2}\right)\right) \partial_{u} \\
X_{6}=h^{\prime}(t) z \partial_{x}+h \partial_{z}+\left(2 h^{\prime \prime}(t) x z+g^{\prime \prime \prime}(t)\left(\frac{z^{3}}{3}+z y^{2}\right)\right) \partial_{u} \\
X_{7}=\alpha \partial_{x}+\left[2 \sigma^{\prime}(t) x+\sigma^{\prime \prime}(t)\left(y^{2}+z^{2}\right)\right] \partial_{u}
\end{gathered}
$$

where $g, h, \sigma$ and $\alpha$ are arbitrary functions of $t$. Ryzhov and Shefter [RS] found some invariant solutions of the equation (1.2), which represent time-dependent flows in a circular or plane Laval nozzle.

First we find that the group invariant solutions with respect to the vector fields $X_{1}-$ $X_{7}$ are polynomial in $x$ with degree $\leq 3$. Then we examine the equations (1.1), (1.2) more closely and observe that this phenomena is essentially caused by the fact that the nonlinear term $u_{x} u_{x x}$ keeps the following polynomial subspace stable:

$$
\mathbb{R}+\mathbb{R} x+\mathbb{R} x^{2}+\mathbb{R} x^{3}
$$

where $\mathbb{R}$ stands for the field of real numbers. This observation suggests a new ansatze of solving the equations (1.1) and (1.2). Since the equation (1.2) contains the Laplace operator $\partial_{y}^{2}+\partial_{z}^{2}$, our approach to (1.2) will involve harmonic analysis and sophisticated integrations. For simplicity, we will solve the equation (1.1) in Section 2 although it can be viewed as a special case of (1.2). Exact solutions of (1.2) will be found in Section 3. 
2. Two-dimensional case. In this section, we study solutions polynomial in $x$ for the Lin-Reissner-Tsien equation (1.1). By comparing the terms of highest degree in $x$, we find that such a solution must be of the form

$$
u=f(t, y)+g(t, y) x+h(t, y) x^{2}+\xi(t, y) x^{3},
$$

where $f(t, y), g(t, y), h(t, y)$ and $\xi(t, y)$ are suitable differentiable functions to be determined. Note that

$$
\begin{gathered}
u_{x}=g+2 h x+3 \xi x^{2}, \quad u_{x x}=2 h+6 \xi x \\
u_{t x}=g_{t}+2 h_{t} x+3 \xi_{t} x^{2}, \quad u_{y y}=f_{y y}+g_{y y} x+h_{y y} x^{2}+\xi_{y y} x^{3} .
\end{gathered}
$$

Now (1.1) becomes

$$
2\left(g_{t}+2 h_{t} x+3 \xi_{t} x^{2}\right)+\left(g+2 h x+3 \xi x^{2}\right)(2 h+6 \xi x)-f_{y y}-g_{y y} x-h_{y y} x^{2}-\xi_{y y} x^{3}=0,
$$

which is equivalent to the following systems of partial differential equations:

$$
\begin{gathered}
\xi_{y y}=18 \xi^{2}, \\
h_{y y}=6 \xi_{t}+18 \xi h, \\
g_{y y}=4 h_{t}+4 h^{2}+6 \xi g, \\
f_{y y}=2 g_{t}+2 g h .
\end{gathered}
$$

First we observe that

$$
\xi=\frac{1}{(\sqrt{3} y+\beta(t))^{2}}
$$

is a solution of the equation (2.5) for any differentiable function $\beta$ of $t$. Substituting (2.9) into (2.6), we get

$$
h_{y y}=-\frac{12 \beta^{\prime}(t)}{(\sqrt{3} y+\beta(t))^{3}}+\frac{18}{(\sqrt{3} y+\beta(t))^{2}} h .
$$

Denote by $\mathbb{Z}$ the ring of integers. Write

$$
h(t, y)=\sum_{i \in \mathbb{Z}} a_{i}(t)(\sqrt{3} y+\beta(t))^{i} .
$$

Then

$$
h_{y y}=\sum_{i \in \mathbb{Z}} 3(i+2)(i+1) a_{i+2}(t)(\sqrt{3} y+\beta(t))^{i} .
$$

Substituting (2.11) and (2.12) into (2.10), we have

$$
\sum_{i \in \mathbb{Z}} 3[(i+2)(i+1)-6] a_{i+2}(t)(\sqrt{3} y+\beta(t))^{i}=-\frac{12 \beta^{\prime}(t)}{(\sqrt{3} y+\beta(t))^{3}} .
$$

Equivalently,

$$
-12 a_{-1}(t)=-12 \beta^{\prime}(t), \quad 3(i+4)(i-1) a_{i+2}(t)=0, \quad i \neq-3 .
$$

Thus

$$
h=\frac{\alpha(t)}{(\sqrt{3} y+\beta(t))^{2}}+\frac{\beta^{\prime}(t)}{\sqrt{3} y+\beta(t)}+\gamma(t)(\sqrt{3} y+\beta(t))^{3},
$$

where $\alpha$ and $\gamma$ are arbitrary differentiable functions of $t$. 
Note that

$$
\begin{aligned}
h_{t}= & -\frac{2 \alpha(t) \beta^{\prime}(t)}{(\sqrt{3} y+\beta(t))^{3}}+\frac{\alpha^{\prime}(t)}{(\sqrt{3} y+\beta(t))^{2}}-\frac{\beta^{\prime}(t)^{2}}{(\sqrt{3} y+\beta(t))^{2}} \\
& +\frac{\beta^{\prime \prime}(t)}{\sqrt{3} y+\beta(t)}+3 \gamma(t) \beta^{\prime}(t)(\sqrt{3} y+\beta(t))^{2}+\gamma^{\prime}(t)(\sqrt{3} y+\beta(t))^{3}
\end{aligned}
$$

and

$$
\begin{aligned}
& h^{2}=\frac{\alpha(t)^{2}}{(\sqrt{3} y+\beta(t))^{4}}+2 \frac{\alpha(t) \beta^{\prime}(t)}{(\sqrt{3} y+\beta(t))^{3}}+\frac{\beta^{\prime}(t)^{2}}{(\sqrt{3} y+\beta(t))^{2}} \\
& +2 \alpha(t) \gamma(t)(\sqrt{3} y+\beta(t))+2 \gamma(t) \beta^{\prime}(t)(\sqrt{3} y+\beta(t))^{2}+\gamma(t)^{2}(\sqrt{3} y+\beta(t))^{6} .
\end{aligned}
$$

Substituting the above two equations into (2.7), we have

$$
\begin{aligned}
& g_{y y}-\frac{6}{(\sqrt{3} y+\beta)^{2}} g=\frac{4 \alpha^{2}}{(\sqrt{3} y+\beta)^{4}}+\frac{4 \alpha^{\prime}}{(\sqrt{3} y+\beta)^{2}}+\frac{4 \beta^{\prime \prime}}{\sqrt{3} y+\beta} \\
& +8 \alpha \gamma(\sqrt{3} y+\beta)+20 \gamma \beta^{\prime}(\sqrt{3} y+\beta)^{2}+4 \gamma^{\prime}(\sqrt{3} y+\beta)^{3}+4 \gamma^{2}(\sqrt{3} y+\beta)^{6} .
\end{aligned}
$$

Write

$$
g(t, y)=\sum_{i \in \mathbb{Z}} b_{i}(t)(\sqrt{3} y+\beta)^{i}
$$

Then

$$
g_{y y}=\sum_{i \in \mathbb{Z}} 3(i+2)(i+1) b_{i+2}(t)(\sqrt{3} y+\beta)^{i} .
$$

Substituting (2.19) and (2.20) into (2.18), we get

$$
\begin{gathered}
3(i+3) i b_{i+2}=0, \quad i \neq-4,-2,-1,1,2,3,6 \\
b_{-2}=\frac{\alpha^{2}}{3}, \quad b_{0}=-\frac{2 \alpha^{\prime}}{3}, \quad b_{1}=-\frac{2 \beta^{\prime \prime}}{3}, \quad b_{3}=\frac{2 \alpha \gamma}{3} \\
b_{4}=\frac{2 \beta^{\prime} \gamma}{3}, \quad b_{5}=\frac{2 \gamma^{\prime}}{27}, \quad b_{8}=\frac{2 \gamma^{2}}{81}
\end{gathered}
$$

Therefore

$$
\begin{aligned}
& g=\frac{\alpha^{2}}{3(\sqrt{3} y+\beta)^{2}}+\frac{\sigma}{\sqrt{3} y+\beta}-\frac{2 \alpha^{\prime}}{3}-\frac{2{\beta^{\prime \prime}}^{\prime}}{3}(\sqrt{3} y+\beta)+\rho(\sqrt{3} y+\beta)^{2} \\
& +\frac{2 \alpha \gamma}{3}(\sqrt{3} y+\beta)^{3}+\frac{2 \beta^{\prime} \gamma}{3}(\sqrt{3} y+\beta)^{4}+\frac{2 \gamma^{\prime}}{27}(\sqrt{3} y+\beta)^{5}+\frac{2 \gamma^{2}}{81}(\sqrt{3} y+\beta)^{8}
\end{aligned}
$$

where $\sigma$ and $\rho$ are arbitrary differentiable functions of $t$. 
Observe that

$$
\begin{aligned}
g_{t} & =-\frac{2 \alpha^{2} \beta^{\prime}}{3(\sqrt{3} y+\beta)^{3}}+\frac{\left(2 \alpha \alpha^{\prime}-3 \sigma \beta^{\prime}\right)}{3(\sqrt{3} y+\beta)^{2}}+\frac{\sigma^{\prime}}{\sqrt{3} y+\beta}-\frac{2 \alpha^{\prime \prime}}{3}-\frac{2 \beta^{\prime} \beta^{\prime \prime}}{3} \\
& +\frac{2\left(3 \rho \beta^{\prime}-\beta^{\prime \prime}\right)}{3}(\sqrt{3} y+\beta)+\left(\rho^{\prime}+2 \alpha \gamma \beta^{\prime}\right)(\sqrt{3} y+\beta)^{2} \\
& +\frac{2 \alpha^{\prime} \gamma+2 \alpha \gamma^{\prime}+8\left(\beta^{\prime}\right)^{2} \gamma}{3}(\sqrt{3} y+\beta)^{3}+\frac{18 \beta^{\prime \prime} \gamma+28 \beta^{\prime} \gamma^{\prime}}{27}(\sqrt{3} y+\beta)^{4} \\
& +\frac{2 \gamma^{\prime \prime}}{27}(\sqrt{3} y+\beta)^{5}+\frac{16 \gamma^{2} \beta^{\prime}}{81}(\sqrt{3} y+\beta)^{7}+\frac{4 \gamma \gamma^{\prime}}{81}(\sqrt{3} y+\beta)^{8}, \\
g h & =\frac{\alpha^{3}}{3(\sqrt{3} y+\beta)^{4}}+\frac{3 \alpha \sigma+\alpha^{2} \beta^{\prime}}{3(\sqrt{3} y+\beta)^{3}}+\frac{3 \beta^{\prime} \sigma-2 \alpha \alpha^{\prime}}{3(\sqrt{3} y+\beta)^{2}}-\frac{2\left(\alpha \beta^{\prime \prime}+\alpha^{\prime} \beta^{\prime}\right)}{3(\sqrt{3} y+\beta)} \\
& +\alpha \rho-\frac{2 \beta^{\prime} \beta^{\prime \prime}}{3}+\left(\alpha^{2} \gamma+\beta^{\prime} \rho\right)(\sqrt{3} y+\beta)+\frac{4 \alpha \beta^{\prime} \gamma+3 \gamma \sigma}{3}(\sqrt{3} y+\beta)^{2} \\
& +\frac{18\left(\beta^{\prime}\right)^{2} \gamma-16 \alpha \gamma^{\prime}}{27}(\sqrt{3} y+\beta)^{3}+\frac{2 \beta^{\prime} \gamma^{\prime}-18 \beta^{\prime \prime} \gamma}{27}(\sqrt{3} y+\beta)^{4}+\gamma \rho(\sqrt{3} y+\beta)^{5} \\
& +\frac{56 \alpha \gamma^{2}}{81}(\sqrt{3} y+\beta)^{6}+\frac{56 \beta^{\prime} \gamma^{2}}{81}(\sqrt{3} y+\beta)^{7}+\frac{2 \gamma^{3}}{81}(\sqrt{3} y+\beta)^{11} .
\end{aligned}
$$

Substituting (2.25) and (2.26) into (2.8), we obtain

$$
\begin{aligned}
& f_{y y}=\frac{2 \alpha^{3}}{3(\sqrt{3} y+\beta)^{4}}+\frac{6 \alpha \sigma-2 \alpha^{2} \beta^{\prime}}{3(\sqrt{3} y+\beta)^{3}}+\frac{6 \sigma^{\prime}-4\left(\alpha \beta^{\prime \prime}+\alpha^{\prime} \beta^{\prime}\right)}{3(\sqrt{3} y+\beta)} \\
& +2 \alpha \rho-\frac{4 \alpha^{\prime \prime}}{3}-\frac{8 \beta^{\prime}{\beta^{\prime \prime}}^{\prime}}{3}+\frac{6 \alpha^{2} \gamma+18 \beta^{\prime} \rho-4{\beta^{\prime \prime}}^{\prime \prime}}{3}(\sqrt{3} y+\beta) \\
& +\frac{20 \alpha \beta^{\prime} \gamma+6 \gamma \sigma+6 \rho^{\prime}}{3}(\sqrt{3} y+\beta)^{2}+\frac{180\left(\beta^{\prime}\right)^{2} \gamma+4 \alpha \gamma^{\prime}+36 \alpha^{\prime} \gamma}{27}(\sqrt{3} y+\beta)^{3} \\
& +\frac{20 \beta^{\prime} \gamma^{\prime}}{9}(\sqrt{3} y+\beta)^{4}+\frac{54 \gamma \rho+4 \gamma^{\prime \prime}}{27}(\sqrt{3} y+\beta)^{5}+\frac{112 \alpha \gamma^{2}}{81}(\sqrt{3} y+\beta)^{6} \\
& +\frac{16 \beta^{\prime} \gamma^{2}}{9}(\sqrt{3} y+\beta)^{7}+\frac{8 \gamma \gamma^{\prime}}{81}(\sqrt{3} y+\beta)^{8}+\frac{4 \gamma^{3}}{81}(\sqrt{3} y+\beta)^{11} .
\end{aligned}
$$

Thus

$$
\begin{aligned}
& f=\frac{\alpha^{3}}{27(\sqrt{3} y+\beta)^{2}}+\frac{3 \alpha \sigma-\alpha^{2} \beta^{\prime}}{9(\sqrt{3} y+\beta)}+\theta+\vartheta y+\alpha \rho y^{2}-\frac{2 \alpha^{\prime \prime}+4 \beta^{\prime}{\beta^{\prime \prime}}^{\prime}}{3} y^{2} \\
& +\frac{1}{9}\left[6 \sigma^{\prime}-4\left(\alpha \beta^{\prime \prime}+\alpha^{\prime} \beta^{\prime}\right)\right](\sqrt{3} y+\beta)[\ln (\sqrt{3} y+\beta)-1]+\frac{3 \alpha^{2} \gamma+9 \beta^{\prime} \rho-2{\beta^{\prime \prime}}^{\prime \prime}}{27}(\sqrt{3} y+\beta)^{3} \\
& +\frac{10 \alpha \beta^{\prime} \gamma+3 \gamma \sigma+3 \rho^{\prime}}{54}(\sqrt{3} y+\beta)^{4}+\frac{45\left(\beta^{\prime}\right)^{2} \gamma+\alpha \gamma^{\prime}+9 \alpha^{\prime} \gamma}{405}(\sqrt{3} y+\beta)^{5} \\
& +\frac{2 \beta^{\prime} \gamma^{\prime}}{81}(\sqrt{3} y+\beta)^{6}+\frac{27 \gamma \rho+2 \gamma^{\prime \prime}}{1701}(\sqrt{3} y+\beta)^{7}+\frac{2 \alpha \gamma^{2}}{243}(\sqrt{3} y+\beta)^{8} \\
& +\frac{2 \beta^{\prime} \gamma^{2}}{243}(\sqrt{3} y+\beta)^{9}+\frac{4 \gamma \gamma^{\prime}}{10935}(\sqrt{3} y+\beta)^{10}+\frac{\gamma^{3}}{9477}(\sqrt{3} y+\beta)^{13},
\end{aligned}
$$

where $\theta$ and $\vartheta$ are arbitrary functions of $t$. 
THEOREM 2.1. We have the following solution of the equation (1.1) blowing up on the surface $\sqrt{3} y+\beta(t)=0$ :

$$
\begin{aligned}
& u=\frac{x^{3}}{(\sqrt{3} y+\beta)^{2}}+\frac{\alpha x^{2}}{(\sqrt{3} y+\beta)^{2}}+\frac{\beta^{\prime} x^{2}}{\sqrt{3} y+\beta}+\gamma(\sqrt{3} y+\beta)^{3} x^{2} \\
& +\left[\frac{\alpha^{2}}{3(\sqrt{3} y+\beta)^{2}}+\frac{\sigma}{\sqrt{3} y+\beta}-\frac{2 \alpha^{\prime}}{3}-\frac{2 \beta^{\prime \prime}}{3}(\sqrt{3} y+\beta)+\rho(\sqrt{3} y+\beta)^{2}\right. \\
& \left.+\frac{2 \alpha \gamma}{3}(\sqrt{3} y+\beta)^{3}+\frac{2 \beta^{\prime} \gamma}{3}(\sqrt{3} y+\beta)^{4}+\frac{2 \gamma^{\prime}}{27}(\sqrt{3} y+\beta)^{5}+\frac{2 \gamma^{2}}{81}(\sqrt{3} y+\beta)^{8}\right] x \\
& +\frac{\alpha^{3}}{27(\sqrt{3} y+\beta)^{2}}+\frac{3 \alpha \sigma-\alpha^{2} \beta^{\prime}}{9(\sqrt{3} y+\beta)}+\theta+\vartheta y+\alpha \rho y^{2}-\frac{2 \alpha^{\prime \prime}+4 \beta^{\prime} \beta^{\prime \prime}}{3} y^{2} \\
& +\frac{1}{9}\left[6 \sigma^{\prime}-4\left(\alpha \beta^{\prime \prime}+\alpha^{\prime} \beta^{\prime}\right)\right](\sqrt{3} y+\beta)[\ln (\sqrt{3} y+\beta)-1]+\frac{3 \alpha^{2} \gamma+9 \beta^{\prime} \rho-2 \beta^{\prime \prime \prime}}{27}(\sqrt{3} y+\beta)^{3} \\
& +\frac{10 \alpha \beta^{\prime} \gamma+3 \gamma \sigma+3 \rho^{\prime}}{54}(\sqrt{3} y+\beta)^{4}+\frac{45\left(\beta^{\prime}\right)^{2} \gamma+\alpha \gamma^{\prime}+9 \alpha^{\prime} \gamma}{405}(\sqrt{3} y+\beta)^{5} \\
& +\frac{2 \beta^{\prime} \gamma^{\prime}}{81}(\sqrt{3} y+\beta)^{6}+\frac{27 \gamma \rho+2 \gamma^{\prime \prime}}{1701}(\sqrt{3} y+\beta)^{7}+\frac{2 \alpha \gamma^{2}}{243}(\sqrt{3} y+\beta)^{8} \\
& +\frac{2 \beta^{\prime} \gamma^{2}}{243}(\sqrt{3} y+\beta)^{9}+\frac{4 \gamma \gamma^{\prime}}{10935}(\sqrt{3} y+\beta)^{10}+\frac{\gamma^{3}}{9477}(\sqrt{3} y+\beta)^{13},
\end{aligned}
$$

where $\alpha, \beta, \gamma, \sigma, \rho, \theta$ and $\vartheta$ are arbitrary functions of $t$, whose derivatives which appeared in the above exist in a certain open set of $\mathbb{R}$.

When $\alpha=\gamma=\sigma=\rho=\theta=\vartheta=0$, the above solution becomes

$$
u=\frac{x^{3}}{(\sqrt{3} y+\beta)^{2}}+\frac{\beta^{\prime} x^{2}}{\sqrt{3} y+\beta}-\frac{2 \beta^{\prime \prime}}{3}(\sqrt{3} y+\beta) x-\frac{4 \beta^{\prime} \beta^{\prime \prime}}{3} y^{2}-\frac{2 \beta^{\prime \prime \prime}}{27}(\sqrt{3} y+\beta)^{3} \text {. }
$$

Take the trivial solution $\xi=0$ of (2.5), which is the only solution polynomial in $y$. Then (2.6) and (2.7) become

$$
h_{y y}=0, \quad g_{y y}=4 h_{t}+4 h^{2} .
$$

Thus

$$
h=\alpha(t)+\beta(t) y
$$

Hence

$$
g_{y y}=4 \alpha^{2}+4 \alpha^{\prime}+4\left(\beta^{\prime}+2 \alpha \beta\right) y+4 \beta^{2} y^{2}
$$

So

$$
g=\gamma+\sigma y+2\left(\alpha^{2}+\alpha^{\prime}\right) y^{2}+\frac{2}{3}\left(\beta^{\prime}+2 \alpha \beta\right) y^{3}+\frac{1}{3} \beta^{2} y^{4}
$$

where $\gamma$ and $\sigma$ are arbitrary functions of $t$. Now (2.8) yields

$$
\begin{aligned}
f_{y y} & =2\left(\alpha \gamma+\gamma^{\prime}\right)+2\left(\alpha \sigma+\beta \gamma+\sigma^{\prime}\right) y+2\left(\beta \sigma+2 \alpha^{3}+6 \alpha \alpha^{\prime}+2 \alpha^{\prime \prime}\right) y^{2} \\
& +\frac{20 \alpha^{2} \beta+12 \alpha \beta^{\prime}+20 \alpha^{\prime} \beta+4 \beta^{\prime \prime}}{3} y^{3}+\frac{10 \alpha \beta^{2}+8 \beta \beta^{\prime}}{3} y^{4}+\frac{2}{3} \beta^{3} y^{5} .
\end{aligned}
$$


Therefore,

$$
\begin{aligned}
f= & \tau+\rho y+\left(\alpha \gamma+\gamma^{\prime}\right) y^{2}+\frac{1}{3}\left(\alpha \sigma+\beta \gamma+\sigma^{\prime}\right) y^{3}+\frac{1}{6}\left(\beta \sigma+2 \alpha^{3}+6 \alpha \alpha^{\prime}+2 \alpha^{\prime \prime}\right) y^{4} \\
& +\frac{5 \alpha^{2} \beta+3 \alpha \beta^{\prime}+5 \alpha^{\prime} \beta+{\beta^{\prime \prime}}^{\prime}}{15} y^{5}+\frac{5 \alpha \beta^{2}+4 \beta \beta^{\prime}}{45} y^{6}+\frac{1}{63} \beta^{3} y^{7} .
\end{aligned}
$$

THEOREM 2.2. The following is a solution of the equation (1.1):

$$
\begin{aligned}
u= & (\alpha+\beta y) x^{2}+\left[\gamma+\sigma y+2\left(\alpha^{2}+\alpha^{\prime}\right) y^{2}+\frac{2}{3}\left(\beta^{\prime}+2 \alpha \beta\right) y^{3}+\frac{1}{3} \beta^{2} y^{4}\right] x \\
& +\tau+\rho y+\left(\alpha \gamma+\gamma^{\prime}\right) y^{2}+\frac{1}{3}\left(\alpha \sigma+\beta \gamma+\sigma^{\prime}\right) y^{3}+\frac{1}{6}\left(\beta \sigma+2 \alpha^{3}+6 \alpha \alpha^{\prime}+2 \alpha^{\prime \prime}\right) y^{4} \\
& +\frac{5 \alpha^{2} \beta+3 \alpha \beta^{\prime}+5 \alpha^{\prime} \beta+\beta^{\prime \prime}}{15} y^{5}+\frac{5 \alpha \beta^{2}+4 \beta \beta^{\prime}}{45} y^{6}+\frac{1}{63} \beta^{3} y^{7},
\end{aligned}
$$

where $\alpha, \beta, \gamma, \sigma, \rho$ and $\tau$ are arbitrary functions of $t$, whose derivatives which appeared in the above exist in a certain open set of $\mathbb{R}$. Moreover, any solution polynomial in $x$ and $y$ of (1.1) must be of the above form. The above solution is smooth (analytic) if all $\alpha, \beta, \gamma, \sigma, \rho$ and $\tau$ are smooth (analytic) functions of $t$.

REMARK 2.3. In addition to the nonzero solution (2.9) of the equation (2.5), the other nonzero solutions are of the form

$$
\xi=\wp_{\iota}(\sqrt{3} y+\beta(t)),
$$

where $\wp_{\iota}(w)$ is the Weierstrass elliptic function such that

$$
\wp_{\iota}^{\prime}(w)^{2}=4\left(\wp_{\iota}(w)^{3}-\iota\right),
$$

and $\iota$ is a nonzero constant and $\beta$ is any function of $t$. When $\beta$ is not a constant, the solutions of (2.6)-(2.8) are extremely complicated. If $\beta$ is constant, we can take $\beta=0$ by adjusting $\iota$. Any solution of (2.6)-(2.8) with $h \neq 0$ is also very complicated. Thus the only simple solution of the equation (1.1) in this case is

$$
u=\wp_{\iota}(\sqrt{3} y) x^{3} .
$$

3. Three-dimensional case. By comparing the terms of highest degree, we find that a solution polynomial in $x$ of the equation (1.2) must be of the form

$$
u=f(t, y, z)+g(t, y, z) x+h(t, y, z) x^{2}+\xi(t, y, z) x^{3},
$$

where $f(t, y, z), g(t, y, z), h(t, y, z)$ and $\xi(t, y, z)$ are suitable differentiable functions to be determined. Note that

$$
\begin{gathered}
u_{x}=g+2 h x+3 \xi x^{2}, \quad u_{x x}=2 h+6 \xi x \\
u_{t x}=g_{t}+2 h_{t} x+3 \xi_{t} x^{2}, \quad u_{y y}=f_{y y}+g_{y y} x+h_{y y} x^{2}+\xi_{y y} x^{3}, \\
u_{z z}=f_{z z}+g_{z z} x+h_{z z} x^{2}+\xi_{z z} x^{3} .
\end{gathered}
$$

Now (1.2) becomes

$$
\begin{aligned}
& 2\left(g_{t}+2 h_{t} x+3 \xi_{t} x^{2}\right)+\left(g+2 h x+3 \xi x^{2}\right)(2 h+6 \xi x)-\left(f_{y y}+f_{z z}\right) \\
& -\left(g_{y y}+g_{z z}\right) x-\left(h_{y y}+h_{z z}\right) x^{2}-\left(\xi_{y y}+\xi_{z z}\right) x^{3}=0,
\end{aligned}
$$


which is equivalent to the following systems of partial differential equations:

$$
\begin{gathered}
\xi_{y y}+\xi_{z z}=18 \xi^{2}, \\
h_{y y}+h_{z z}=6 \xi_{t}+18 \xi h, \\
g_{y y}+g_{z z}=4 h_{t}+4 h^{2}+6 \xi g, \\
f_{y y}+f_{z z}=2 h g_{t}+2 g h .
\end{gathered}
$$

First we observe that

$$
\xi=\frac{1}{(\sqrt{3}(y \cos \alpha(t)+z \sin \alpha(t))+\beta(t))^{2}}
$$

is a solution of the equation (3.6), where $\alpha$ and $\beta$ are suitable differentiable functions of $t$. With the above $\xi,(3.7)$ becomes

$$
h_{y y}+h_{z z}=-\frac{12\left(\sqrt{3} \alpha^{\prime}(-y \sin \alpha+z \cos \alpha)+\beta^{\prime}\right)}{(\sqrt{3}(y \cos \alpha+z \sin \alpha)+\beta)^{3}}+\frac{18 h}{(\sqrt{3}(y \cos \alpha+z \sin \alpha)+\beta)^{2}} \text {. }
$$

In order to solve (3.11), we change variables:

$$
\zeta=\sqrt{3}(\cos \alpha y+\sin \alpha z)+\beta, \quad \eta=\sqrt{3}(-\sin \alpha y+\cos \alpha z) .
$$

Then

$$
\partial_{y}=\sqrt{3}\left(\cos \alpha \partial_{\zeta}-\sin \alpha \partial_{\eta}\right), \quad \partial_{z}=\sqrt{3}\left(\sin \alpha \partial_{\zeta}+\cos \alpha \partial_{\eta}\right)
$$

Thus

$$
\partial_{y}^{2}+\partial_{z}^{2}=3\left(\cos \alpha \partial_{\zeta}-\sin \alpha \partial_{\eta}\right)^{2}+3\left(\sin \alpha \partial_{\zeta}+\cos \alpha \partial_{\eta}\right)^{2}=3\left(\partial_{\zeta}^{2}+\partial_{\eta}^{2}\right)
$$

Note that

$$
\partial_{t}(\zeta)=\alpha^{\prime} \eta+\beta^{\prime}, \quad \partial_{t}(\eta)=\alpha^{\prime}(\beta-\zeta) .
$$

The equation (3.11) can be rewritten as

$$
h_{\zeta \zeta}+h_{\eta \eta}=-4\left(\alpha^{\prime} \eta+\beta^{\prime}\right) \zeta^{-3}+6 \zeta^{-2} h .
$$

In order to solve the above equation, we assume

$$
h=\sum_{i \in \mathbb{Z}} a_{i}(t, \eta) \zeta^{i} .
$$

Now (3.16) becomes

$$
\sum_{i \in \mathbb{Z}} a_{i \eta \eta} \zeta^{i}+\sum_{i \in \mathbb{Z}} i(i-1) a_{i} \zeta^{i-2}=-4\left(\alpha^{\prime} \eta+\beta^{\prime}\right) \zeta^{-3}+6 \sum_{i \in \mathbb{Z}} a_{i} \zeta^{i-2},
$$

which is equivalent to

$$
a_{-3 \eta \eta}+2 a_{-1}=-4\left(\alpha^{\prime} \eta+\beta^{\prime}\right)+6 a_{-1}, \quad a_{i \eta \eta}+(i+2)(i+1) a_{i+2}=6 a_{i+2}
$$

for $-3 \neq i \in \mathbb{Z}$. Hence

$$
a_{-1}=\frac{1}{4} a_{-3 \eta \eta}+\alpha^{\prime} \eta+\beta^{\prime}, \quad(i+4)(i-1) a_{i+2}=-a_{i \eta \eta} \quad \text { for } \quad-3 \neq i \in \mathbb{Z} .
$$

When $i=-4$ and $i=1$, we get $a_{-4 \eta \eta}=a_{1 \eta \eta}=0$. Moreover, $a_{-2}$ and $a_{3}$ can be any functions. 
Take

$$
\begin{gathered}
a_{3}=\sigma, \quad a_{-2}=\rho, \quad a_{-1}=\alpha^{\prime} \eta+\beta^{\prime}, \\
a_{1}=a_{-1-2 i}=a_{-2-2 i}=0 \quad \text { for } 0<i \in \mathbb{Z}
\end{gathered}
$$

in order to avoid an infinite number of negative powers of $\zeta$ in (3.17), where $\sigma$ and $\rho$ are functions of $t$ and $\eta$ differentiable in a certain domain. By (3.20),

$$
\begin{gathered}
a_{3+2 k}=\frac{(-1)^{k} \partial_{\eta}^{2 k}(\sigma)}{\prod_{i=1}^{k}(2 i+5)(2 i)}=\frac{(-1)^{k} 15 \partial_{\eta}^{2 k}(\sigma)}{(2 k+5)(2 k+3)(2 k+1) !}, \\
a_{-2+2 k}=\frac{(-1)^{k} \partial_{\eta}^{2 k}(\rho)}{\prod_{i=1}^{k}(2 i)(2 i-5)}=\frac{(-1)^{k}(2 k-1)(2 k-3) \partial_{\eta}^{2 k}(\rho)}{3(2 k) !} .
\end{gathered}
$$

Therefore,

$$
\begin{aligned}
h & =\left(\alpha^{\prime} \eta+\beta^{\prime}\right) \zeta^{-1}+\sum_{k=0}^{\infty}(-1)^{k}\left[\frac{15 \partial_{\eta}^{2 k}(\sigma) \zeta^{3}}{(2 k+5)(2 k+3)(2 k+1) !}\right. \\
& \left.+\frac{(2 k-1)(2 k-3) \partial_{\eta}^{2 k}(\rho) \zeta^{-2}}{3(2 k) !}\right] \zeta^{2 k}
\end{aligned}
$$

is a solution of (3.16).

By (3.12) and (3.14), (3.8) is equivalent to

$$
g_{\zeta \zeta}+g_{\eta \eta}=\frac{4}{3} h_{t}+\frac{4}{3} h^{2}+2 \zeta^{-2} g .
$$

Note that

$$
\begin{aligned}
& h_{t}=\left(\alpha^{\prime \prime} \eta+\beta^{\prime \prime}+\left(\alpha^{\prime}\right)^{2} \beta\right) \zeta^{-1}-\left(\alpha^{\prime}\right)^{2}-\left(\alpha^{\prime} \eta+\beta^{\prime}\right)^{2} \zeta^{-2}+\sum_{k=0}^{\infty}(-1)^{k} \zeta^{2 k} \\
& \times\left(\frac{15 \partial_{\eta}^{2 k}\left(\sigma_{t}+\alpha^{\prime}(\beta-\zeta) \sigma_{\eta}\right) \zeta^{3}}{(2 k+5)(2 k+3)(2 k+1) !}+\frac{(2 k-1)(2 k-3) \partial_{\eta}^{2 k}\left(\rho_{t}+\alpha^{\prime}(\beta-\zeta) \rho_{\eta}\right) \zeta^{-2}}{3(2 k) !}\right) \\
& +\left(\alpha^{\prime} \eta+\beta^{\prime}\right)\left[\frac{15 \partial_{\eta}^{2 k}(\sigma) \zeta^{2}}{(2 k+5)(2 k+1) !}+\frac{(2 k-1)(2 k-2)(2 k-3) \partial_{\eta}^{2 k}(\rho) \zeta^{-3}}{3(2 k) !}\right] .
\end{aligned}
$$

For convenience of solving the equation (3.26), we denote

$$
\frac{4}{3} h_{t}+\frac{4}{3} h^{2}=\sum_{i=-4}^{\infty} b_{i}(t, \eta) \zeta^{i}
$$

by (3.25) and (3.27). In particular,

$$
\begin{gathered}
b_{-4}=\frac{4}{3} \rho^{2}, \quad b_{-3}=0 \\
b_{-2}=\frac{4}{3}\left(\rho_{t}+\alpha^{\prime} \beta \rho_{\eta}\right)+\frac{4}{9} \rho_{\eta \eta} \rho \\
b_{-1}=\frac{4}{3}\left[\alpha^{\prime \prime} \eta+\beta^{\prime \prime}+\left(\alpha^{\prime}\right)^{2} \beta-\alpha^{\prime} \rho_{\eta}\right]+\frac{4}{9}\left(\alpha^{\prime} \eta+\beta^{\prime}\right) \rho_{\eta \eta}, \\
b_{0}=-\frac{4\left(\alpha^{\prime}\right)^{2}}{3}+\frac{2}{9}\left(\rho_{t \eta \eta}+\alpha^{\prime} \beta \rho_{\eta \eta \eta}\right)+\frac{1}{9} \partial_{\eta}^{4}(\rho) \rho+\frac{1}{27} \rho_{\eta \eta}^{2} .
\end{gathered}
$$


Suppose that

$$
g=\sum_{i \in \mathbb{Z}} c_{i}(t, \eta) \zeta^{i}
$$

is a solution of (3.26). Then

$$
\sum_{i \in \mathbb{Z}}\left[i(i-1) c_{i} \zeta^{i-2}+c_{i \eta \eta} \zeta^{i}\right]=\sum_{r=-4}^{\infty} b_{r} \zeta^{r}+\sum_{i \in \mathbb{Z}} 2 c_{i} \zeta^{i-2}
$$

Equivalently,

$$
(i+3) i c_{i+2}=b_{i}-c_{i \eta \eta}, \quad(r+3) r c_{r+2}=-c_{r \eta \eta}, \quad r<-4 \leq i .
$$

By the second equation in (3.35), we take

$$
c_{r}=0 \quad \text { for } r<-4
$$

to avoid an infinite number of negative powers of $\zeta$ in (3.33). Letting $i=-3,0$, we get

$$
b_{-3}=c_{-3 \eta \eta}, \quad b_{0}=c_{0 \eta \eta}
$$

The first equation is naturally satisfied because $c_{-3}=-c_{-5 \eta \eta} / 10=0$. Taking $i=$ $-2,-4$ and $r=-6$ in $(3.35)$, we obtain

$$
c_{0}=\frac{1}{2} c_{-2 \eta \eta}-\frac{1}{2} b_{-2}, \quad c_{-2}=\frac{1}{4} b_{-4} .
$$

So

$$
c_{0}=\frac{1}{8} \partial_{\eta}^{2}\left(b_{-4}\right)-\frac{1}{2} b_{-2}
$$

Thus we get a constraint:

$$
b_{0}=\frac{1}{8} \partial_{\eta}^{4}\left(b_{-4}\right)-\frac{1}{2} \partial_{\eta}^{2}\left(b_{-2}\right)
$$

Equivalently,

$$
\begin{aligned}
& -\frac{4\left(\alpha^{\prime}\right)^{2}}{3}+\frac{2}{9}\left(\rho_{t \eta \eta}+\alpha^{\prime} \beta \rho_{\eta \eta \eta}\right)+\frac{1}{9} \partial_{\eta}^{4}(\rho) \rho+\frac{1}{27} \rho_{\eta \eta}^{2} \\
& =\frac{1}{6} \partial_{\eta}^{4}\left(\rho^{2}\right)-\frac{2}{3}\left(\rho_{t \eta \eta}+\alpha^{\prime} \beta \rho_{\eta \eta \eta}\right)-\frac{2}{9} \partial_{\eta}^{2}\left(\rho_{\eta \eta} \rho\right) .
\end{aligned}
$$

Thus

$$
48\left(\rho_{t \eta \eta}+\alpha^{\prime} \beta \rho_{\eta \eta \eta}\right)+6 \partial_{\eta}^{4}(\rho) \rho+2 \rho_{\eta \eta}^{2}-9 \partial_{\eta}^{4}\left(\rho^{2}\right)+12 \partial_{\eta}^{2}\left(\rho_{\eta \eta} \rho\right)=72\left(\alpha^{\prime}\right)^{2} .
$$


It can be proved by considering the terms of highest degree that any solution of (3.42) polynomial in $\eta$ must be of the form

$$
\rho=\gamma_{0}(t)+\gamma_{1}(t) \eta+\gamma_{2}(t) \eta^{2}
$$

Then (3.42) becomes

$$
12 \gamma_{2}^{\prime}-20 \gamma_{2}^{2}=9\left(\alpha^{\prime}\right)^{2}
$$

So

$$
\alpha^{\prime}=\frac{2 \epsilon}{3} \sqrt{3 \gamma_{2}^{\prime}-5 \gamma_{2}^{2}} \Rightarrow \alpha=\frac{2 \epsilon}{3} \int \sqrt{3 \gamma_{2}^{\prime}-5 \gamma_{2}^{2}} d t
$$

where $\epsilon= \pm 1$. Replacing $\beta$ by $-\beta$ if necessary, we can take $\epsilon=1$. Under the assumption $(3.43)$,

$$
h=\rho \zeta^{-2}+\left(\alpha^{\prime} \eta+\beta^{\prime}\right) \zeta^{-1}+\frac{\gamma_{2}}{6}+\sum_{k=0}^{\infty}(-1)^{k} \frac{15 \partial_{\eta}^{2 k}(\sigma) \zeta^{3+2 k}}{(2 k+5)(2 k+3)(2 k+1) !}
$$

and

$$
\begin{gathered}
b_{-2}=\frac{4}{3}\left(\rho_{t}+\alpha^{\prime} \beta \rho_{\eta}\right)+\frac{8}{9} \gamma_{2} \rho \\
b_{-1}=\frac{4}{3}\left[\alpha^{\prime \prime} \eta+\beta^{\prime \prime}+\left(\alpha^{\prime}\right)^{2} \beta-\alpha^{\prime} \rho_{\eta}\right]+\frac{8}{9}\left(\alpha^{\prime} \eta+\beta^{\prime}\right) \gamma_{2}, \\
b_{0}=-\frac{4\left(\alpha^{\prime}\right)^{2}}{3}+\frac{4}{9} \gamma_{2}^{\prime}+\frac{4}{27} \gamma_{2}^{2}
\end{gathered}
$$

by $(3.30)-(3.32)$.

Denote

$$
\Psi_{\langle\beta, \rho, \sigma\rangle}(t, \eta, \zeta)=\sum_{i=1}^{\infty} b_{i} \zeta^{i}
$$

For any real function $F(t, \eta)$ analytic at $\eta=\eta_{0}$, we define

$$
F\left(t, \eta_{0}+\sqrt{-1} \zeta\right)=\sum_{r=0}^{\infty} \frac{\partial_{\eta}^{r}(F)\left(t, \eta_{0}\right)}{r !}(\sqrt{-1} \zeta)^{r}
$$


Note that

$$
\begin{aligned}
& \sum_{k=0}^{\infty}(-1)^{k} \frac{15 \partial_{\eta}^{2 k}(\sigma) \zeta^{3+2 k}}{(2 k+5)(2 k+3)(2 k+1) !}=15 \zeta^{2} \int_{0}^{\zeta}\left(\sum_{k=0}^{\infty}(-1)^{k} \frac{\partial_{\eta}^{2 k}(\sigma) \tau_{1}^{2 k}}{(2 k+5)(2 k+3)(2 k) !}\right) d \tau_{1} \\
= & 15 \int_{0}^{\zeta} \tau_{2} \int_{0}^{\tau_{2}}\left(\sum_{k=0}^{\infty}(-1)^{k} \frac{\partial_{\eta}^{2 k}(\sigma) \tau_{1}^{2 k}}{(2 k+5)(2 k) !}\right) d \tau_{1} d \tau_{2} \\
= & 15 \zeta^{-2} \int_{0}^{\zeta} \tau_{3} \int_{0}^{\tau_{3}} \tau_{2} \int_{0}^{\tau_{2}}\left(\sum_{k=0}^{\infty}(-1)^{k} \frac{\partial_{\eta}^{2 k}(\sigma) \tau_{1}^{2 k}}{(2 k) !}\right) d \tau_{1} d \tau_{2} d \tau_{3} \\
& =\frac{15}{2} \zeta^{-2} \int_{0}^{\zeta} \tau_{3} \int_{0}^{\tau_{3}} \tau_{2} \int_{0}^{\tau_{2}}\left[\sigma\left(t, \eta+\sqrt{-1} \tau_{1}\right)+\sigma\left(t, \eta-\sqrt{-1} \tau_{1}\right)\right] d \tau_{1} d \tau_{2} d \tau_{3}, \\
& \partial_{t}\left[\sum_{k=0}^{\infty}(-1)^{k} \frac{15 \partial_{\eta}^{2 k}(\sigma) \zeta^{3+2 k}}{(2 k+5)(2 k+3)(2 k+1) !}\right] \\
= & \sum_{k=0}^{\infty}(-1)^{k} \frac{15 \partial_{\eta}^{2 k}\left(\sigma_{t}\right) \zeta^{3+2 k}}{(2 k+5)(2 k+3)(2 k+1) !}+\alpha^{\prime} \beta \sum_{k=0}^{\infty}(-1)^{k} \frac{15 \partial_{\eta}^{2 k+1}(\sigma) \zeta^{3+2 k}}{(2 k+5)(2 k+3)(2 k+1) !} \\
& -\alpha^{\prime} \sum_{k=0}^{\infty}(-1)^{k} \frac{15 \partial_{\eta}^{2 k+1}(\sigma) \zeta^{4+2 k}}{(2 k+5)(2 k+3)(2 k+1) !}+\left(\alpha^{\prime} \eta+\beta^{\prime}\right) \sum_{k=0}^{\infty}(-1)^{k} \frac{15 \partial_{\eta}^{2 k}(\sigma) \zeta^{2+2 k}}{(2 k+5)(2 k+1) !} \\
& =\frac{15}{2} \zeta^{-2} \int_{0}^{\zeta} \tau_{3}^{\tau_{3}} \int_{0}^{\tau_{2}} \int_{0}^{\tau_{2}}\left[\sigma_{t}\left(t, \eta+\sqrt{-1} \tau_{1}\right)+\sigma_{t}\left(t, \eta-\sqrt{-1} \tau_{1}\right)\right] d \tau_{1} d \tau_{2} d \tau_{3} \\
& +\frac{15 \alpha^{\prime}(\zeta-\beta)}{2 \zeta^{2}} \sqrt{-1} \int_{0}^{\zeta} \tau_{2} \int_{0}^{\tau_{2}} \tau_{1}\left[\sigma\left(t, \eta+\sqrt{-1} \tau_{1}\right)-\sigma\left(t, \eta-\sqrt{-1} \tau_{1}\right)\right] d \tau_{1} d \tau_{2} \\
& \left.\frac{15}{2} \eta+\beta^{\prime}\right) \zeta^{-3} \int_{0}^{\zeta} \tau_{2}^{3} \int_{0}^{\tau_{2}}\left[\sigma\left(t, \eta+\sqrt{-1} \tau_{1}\right)+\sigma\left(t, \eta-\sqrt{-1} \tau_{1}\right)\right] d \tau_{1} d \tau_{2} .
\end{aligned}
$$

According to (3.28) and (3.50), we have

$$
\begin{aligned}
& \Psi_{\langle\beta, \rho, \sigma\rangle}(t, \eta, \zeta)= \\
& 75 \zeta^{-4}\left(\int_{0}^{\zeta} \tau_{3} \int_{0}^{\tau_{3}} \tau_{2} \int_{0}^{\tau_{2}}\left[\sigma\left(t, \eta+\sqrt{-1} \tau_{1}\right)+\sigma\left(t, \eta-\sqrt{-1} \tau_{1}\right)\right] d \tau_{1} d \tau_{2} d \tau_{3}\right)^{2} \\
& +10 \zeta^{-2} \int_{0}^{\zeta} \tau_{3} \int_{0}^{\tau_{3}} \tau_{2} \int_{0}^{\tau_{2}}\left[\sigma_{t}\left(t, \eta+\sqrt{-1} \tau_{1}\right)+\sigma_{t}\left(t, \eta-\sqrt{-1} \tau_{1}\right)\right] d \tau_{1} d \tau_{2} d \tau_{3} \\
& +\frac{10 \alpha^{\prime}(\zeta-\beta)}{\zeta^{2}} \sqrt{-1} \int_{0}^{\zeta} \tau_{2} \int_{0}^{\tau_{2}} \tau_{1}\left[\sigma\left(t, \eta+\sqrt{-1} \tau_{1}\right)-\sigma\left(t, \eta-\sqrt{-1} \tau_{1}\right)\right] d \tau_{1} d \tau_{2} \\
& +10\left(\alpha^{\prime} \eta+\beta^{\prime}\right) \zeta^{-3} \int_{0}^{\zeta} \tau_{2}^{3} \int_{0}^{\tau_{2}}\left[\sigma\left(t, \eta+\sqrt{-1} \tau_{1}\right)+\sigma\left(t, \eta-\sqrt{-1} \tau_{1}\right)\right] d \tau_{1} d \tau_{2} \\
& +20\left(\int_{0}^{\zeta} \tau_{3} \int_{0}^{\tau_{3}} \tau_{2} \int_{0}^{\tau_{2}}\left[\sigma\left(t, \eta+\sqrt{-1} \tau_{1}\right)+\sigma\left(t, \eta-\sqrt{-1} \tau_{1}\right)\right] d \tau_{1} d \tau_{2} d \tau_{3}\right) \\
& \times \zeta^{-2}\left(\rho \zeta^{-2}+\left(\alpha^{\prime} \eta+\beta^{\prime}\right) \zeta^{-1}+\frac{\gamma_{2}}{6}\right) .
\end{aligned}
$$


Now

$$
c_{-2}=\frac{\rho^{2}}{3}
$$

by (3.29) and (3.38). According to (3.35) with $i=-3,0, c_{-1}$ and $c_{2}$ can be arbitrary. For convenience, we redenote

$$
c_{-1}=\kappa(t, \eta), \quad c_{2}=\omega(t, \eta) .
$$

Moreover, (3.29), (3.39) and (3.47) imply

$$
c_{0}=\frac{\rho_{\eta}^{2}}{3}-\frac{2}{3}\left(\rho_{t}+\alpha^{\prime} \beta \rho_{\eta}\right)+\frac{2}{9} \gamma_{2} \rho .
$$

Furthermore, by (3.31) and (3.35),

$$
c_{1}=\frac{\kappa_{\eta \eta}}{2}-\frac{2}{3}\left[\alpha^{\prime \prime} \eta+\beta^{\prime \prime}+\left(\alpha^{\prime}\right)^{2} \beta-\alpha^{\prime} \rho_{\eta}\right]-\frac{4}{9}\left(\alpha^{\prime} \eta+\beta^{\prime}\right) \gamma_{2} .
$$

In addition, (3.35) and (3.56) yield

$$
\begin{gathered}
c_{2 k+3}=\frac{(-1)^{k+1} \partial_{\eta}^{2 k+4}(\kappa)}{2(k+2)(2 k+2) !}+\sum_{i=0}^{k} \frac{(-1)^{k-i}(i+1)(2 i) !}{(k+2)(2 k+2) !} \partial_{\eta}^{2(k-i)}\left(b_{2 i+1}\right), \\
c_{2 k+4}=\frac{(-1)^{k+1} 3 \partial_{\eta}^{2 k+2}(\omega)}{(2 k+5)(2 k+3) !}+\sum_{i=0}^{k} \frac{(-1)^{k-i}(2 i+3)(2 i+1) !}{(2 k+5)(2 k+3) !} \partial_{\eta}^{2(k-i)}\left(b_{2 i+2}\right)
\end{gathered}
$$

for $0 \leq k \in \mathbb{Z}$.

Set

$$
\begin{aligned}
& \Phi_{\langle\beta, \rho, \sigma, \kappa, \omega\rangle}(t, \eta, \zeta)=\kappa \zeta^{-1}+\frac{\kappa_{\eta \eta} \zeta}{2}+\omega \zeta^{2}+\sum_{i=3}^{\infty} c_{i} \zeta^{i} \\
& =-\zeta \partial_{\zeta} \zeta^{-1}\left[\sum_{k=0}^{\infty}(-1)^{k} \frac{\partial_{\eta}^{2 k}(\kappa) \zeta^{2 k}}{(2 k) !}\right]+\zeta^{2} \sum_{k=0}^{\infty}(-1)^{k} \frac{3 \partial_{\eta}^{2 k}(\omega) \zeta^{2 k}}{(2 k+3)(2 k+1) !} \\
& +\sum_{k=0}^{\infty} \sum_{i=0}^{k} \frac{(-1)^{k-i}(i+1)(2 i) !}{(k+2)(2 k+2) !} \partial_{\eta}^{2(k-i)}\left(b_{2 i+1}\right) \zeta^{2 k+3} \\
& +\sum_{k=0}^{\infty} \sum_{i=0}^{k} \frac{(-1)^{k-i}(2 i+3)(2 i+1) !}{(2 k+5)(2 k+3) !} \partial_{\eta}^{2(k-i)}\left(b_{2 i+2}\right) \zeta^{2 k+4} .
\end{aligned}
$$

Note that

$$
\begin{aligned}
& \zeta^{2} \sum_{k=0}^{\infty}(-1)^{k} \frac{3 \partial_{\eta}^{2 k}(\omega) \zeta^{2 k}}{(2 k+3)(2 k+1) !} \\
= & \frac{3}{2} \zeta^{-1} \int_{0}^{\zeta} \tau_{2} \int_{0}^{\tau_{2}}\left[\omega\left(t, \eta+\sqrt{-1} \tau_{1}\right)+\omega\left(t, \eta-\sqrt{-1} \tau_{1}\right)\right] d \tau_{1} d \tau_{2} .
\end{aligned}
$$

Moreover,

$$
\Psi_{\langle\beta, \rho, \sigma\rangle}(t, \eta, 0)=0, \quad b_{i}=\frac{\partial_{\zeta}^{i}\left(\Psi_{\langle\beta, \rho, \sigma\rangle}\right)(t, \eta, 0)}{i !} \quad \text { for } 0<i \in \mathbb{Z} .
$$


Thus

$$
\begin{aligned}
& \Phi_{\langle\beta, \rho, \sigma, \kappa, \omega\rangle}(t, \eta, \zeta)=\frac{3}{2} \zeta^{-1} \int_{0}^{\zeta} \tau_{2} \int_{0}^{\tau_{2}}\left[\omega\left(t, \eta+\sqrt{-1} \tau_{1}\right)+\omega\left(t, \eta-\sqrt{-1} \tau_{1}\right)\right] d \tau_{1} d \tau_{2} \\
& -\frac{1}{2} \zeta \partial_{\zeta} \zeta^{-1}\left[\kappa\left(t, \eta+\sqrt{-1} \tau_{1}\right)+\kappa\left(t, \eta-\sqrt{-1} \tau_{1}\right)\right] \\
& +\sum_{k=0}^{\infty} \sum_{i=0}^{k} \frac{(-1)^{k-i}(i+1) \partial_{\eta}^{2(k-i)} \partial_{\zeta}^{2 i+1}\left(\Psi_{\langle\beta, \rho, \sigma\rangle}\right)(t, \eta, 0)}{(2 i+1)(k+2)(2 k+2) !} \zeta^{2 k+3} \\
& +\sum_{k=0}^{\infty} \sum_{i=0}^{k} \frac{(-1)^{k-i}(2 i+3) \partial_{\eta}^{2(k-i)} \partial_{\zeta}^{2 i+2}\left(\Psi_{\langle\beta, \rho, \sigma\rangle}\right)(t, \eta, 0)}{(2 i+2)(2 k+5)(2 k+3) !} \zeta^{2 k+4}
\end{aligned}
$$

in which the summations are finite if $\sigma(t, \eta)$ is polynomial in $\eta$. Now

$$
\begin{aligned}
h & =\rho \zeta^{-2}+\left(\alpha^{\prime} \eta+\beta^{\prime}\right) \zeta^{-1}+\frac{\gamma_{2}}{6}+\frac{15}{2} \zeta^{-2} \\
& \times \int_{0}^{\zeta} \tau_{3} \int_{0}^{\tau_{3}} \tau_{2} \int_{0}^{\tau_{2}}\left[\sigma\left(t, \eta+\sqrt{-1} \tau_{1}\right)+\sigma\left(t, \eta-\sqrt{-1} \tau_{1}\right)\right] d \tau_{1} d \tau_{2} d \tau_{3}, \\
g & =\Phi_{\langle\beta, \rho, \sigma, \kappa, \omega\rangle}(t, \eta, \zeta)+\frac{\rho^{2}}{3} \zeta^{-2}+\frac{\rho_{\eta}^{2}}{3}-\frac{2}{3}\left(\rho_{t}+\alpha^{\prime} \beta \rho_{\eta}\right)+\frac{2}{9} \gamma_{2} \rho \\
& -\frac{2}{3}\left[\alpha^{\prime \prime} \eta+\beta^{\prime \prime}+\left(\alpha^{\prime}\right)^{2} \beta-\alpha^{\prime} \rho_{\eta}\right] \zeta-\frac{4}{9}\left(\alpha^{\prime} \eta+\beta^{\prime}\right) \gamma_{2} \zeta .
\end{aligned}
$$

Denote

$$
\Lambda_{\langle\beta, \rho, \sigma, \kappa, \omega\rangle}(t, \eta, \zeta)=\frac{2}{3}\left(g h+\partial_{t}(g)\right)
$$

Then the equation (3.9) becomes

$$
f_{\eta \eta}+f_{\zeta \zeta}=\Lambda_{\langle\beta, \rho, \sigma, \kappa, \omega\rangle}(t, \eta, \zeta)
$$

Set

$$
w=\frac{\eta+\sqrt{-1} \zeta}{2}, \quad \bar{w}=\frac{\eta-\sqrt{-1} \zeta}{2} .
$$

Then

$$
\partial_{\eta}=\frac{1}{2}\left(\partial_{w}+\partial_{\bar{w}}\right), \quad \partial_{\zeta}=\frac{\sqrt{-1}}{2}\left(\partial_{w}-\partial_{\bar{w}}\right)
$$

Hence

$$
\partial_{\eta}^{2}+\partial_{\zeta}^{2}=\partial_{w} \partial_{\bar{w}}
$$

A complex function

$$
G(\mu) \text { is called bar-homomorphic if } \overline{G(\mu)}=G(\bar{\mu}) .
$$

For instance, trigonometric functions, polynomials with real coefficients and elliptic functions with bar-invariant periods are bar-homomorphic functions. The extended function 
$F(t, \mu)$ in $(3.51)$ is bar-homomorphic in $\mu$. Now we have

$$
\begin{aligned}
f= & \int_{w_{0}}^{w} \int_{\overline{w_{0}}}^{\bar{w}} \Lambda_{\langle\beta, \rho, \sigma, \kappa, \omega\rangle}(t, \mu+\bar{\mu}, \sqrt{-1}(\bar{\mu}-\mu)) d \bar{\mu} d \mu+\chi(t, \eta+\sqrt{-1} \zeta) \\
& +\chi(t, \eta-\sqrt{-1} \zeta)+\sqrt{-1}[\nu(t, \eta+\sqrt{-1} \zeta)-\nu(t, \eta-\sqrt{-1} \zeta)]
\end{aligned}
$$

where $w_{0}$ is a fixed complex number and $\chi(t, \mu), \nu(t, \mu)$ are complex functions in real variable $t$ and bar-homomorphic in complex variable $\mu$.

THEOREM 3.1. In terms of the notions in (3.12), the function $u=\zeta^{-2} x^{3}+h x^{2}+g x+f$ is a solution of the equation (1.2) blowing up on the hypersurface $\sqrt{3}(\cos \alpha y+\sin \alpha z)+\beta=0$, with $h$ given in (3.65), g given in (3.66) via (3.54) and (3.64), and $f$ given in (3.73) via (3.67). The involved parametric function $\rho$ is given in (3.43), $\alpha$ is given in (3.45), and $\sigma, \kappa, \omega$ are real functions in real variable $t$ and $\eta$.

Next we want to find a more explicit formula when $\sigma=\kappa=\omega=\chi=\nu=0$. In this case,

$$
h=\rho \zeta^{-2}+\left(\alpha^{\prime} \eta+\beta^{\prime}\right) \zeta^{-1}+\frac{\gamma_{2}}{6}
$$

and

$$
\begin{aligned}
g= & \frac{\rho^{2}}{3} \zeta^{-2}+\frac{\rho_{\eta}^{2}}{3}-\frac{2}{3}\left(\rho_{t}+\alpha^{\prime} \beta \rho_{\eta}\right)+\frac{2}{9} \gamma_{2} \rho \\
& -\frac{2}{3}\left[\alpha^{\prime \prime} \eta+\beta^{\prime \prime}+\left(\alpha^{\prime}\right)^{2} \beta-\alpha^{\prime} \rho_{\eta}\right] \zeta-\frac{4}{9}\left(\alpha^{\prime} \eta+\beta^{\prime}\right) \gamma_{2} \zeta .
\end{aligned}
$$

Moreover, (3.15) and (3.43) yield

$$
\begin{aligned}
& g_{t}+h g=\frac{\rho^{3}}{3} \zeta^{-4}-\frac{\left(\alpha^{\prime} \eta+\beta^{\prime}\right) \rho^{2}}{3} \zeta^{-3}+\frac{5 \gamma_{2} \rho^{2}+6 \rho \rho_{\eta}^{2}}{18} \zeta^{-2}+\left[\frac { \alpha ^ { \prime } \eta + \beta ^ { \prime } } { 9 } \left(3 \rho_{\eta}^{2}\right.\right. \\
& \left.\left.-6 \rho_{t}-6 \alpha^{\prime} \beta \rho_{\eta}-2 \gamma_{2} \rho\right)-\frac{2}{3} \rho\left(\alpha^{\prime \prime} \eta+\beta^{\prime \prime}+\left(\alpha^{\prime}\right)^{2} \beta\right)\right] \zeta^{-1}+\frac{2 \rho_{\eta} \rho_{t \eta}}{3}+\frac{2 \gamma_{2}^{\prime} \rho}{9} \\
& -\frac{2}{3}\left(\rho_{t t}+2 \alpha^{\prime} \beta \rho_{t \eta}+\left(\alpha^{\prime} \beta\right)^{\prime} \rho_{\eta}+2\left(\alpha^{\prime} \beta\right)^{2} \gamma_{2}\right)+\frac{\gamma_{2} \rho_{t}}{9}+\frac{13 \alpha^{\prime} \beta \gamma_{2} \rho_{\eta}}{9}+\frac{\gamma_{2} \rho_{\eta}^{2}}{18}+\frac{\gamma_{2}^{2} \rho}{27} \\
& -\frac{4}{3}\left(\alpha^{\prime} \eta+\beta^{\prime}\right)\left(\alpha^{\prime \prime} \eta+\beta^{\prime \prime}+\left(\alpha^{\prime}\right)^{2} \beta-\alpha^{\prime} \rho_{\eta}\right)-\frac{8}{9} \gamma_{2}\left(\alpha^{\prime} \eta+\beta^{\prime}\right)^{2}+\left[\frac{2 \alpha^{\prime} \rho_{t \eta}}{3}\right. \\
& +\frac{\gamma_{2}}{9}\left(19\left(\alpha^{\prime}\right)^{2} \beta-5 \alpha^{\prime \prime} \eta-5 \beta^{\prime \prime}\right)-\frac{13 \alpha^{\prime} \gamma_{2} \rho_{\eta}}{9}-\frac{2}{27} \gamma_{2}^{2}\left(\alpha^{\prime} \eta+\beta^{\prime}\right)-\frac{2}{3}\left(\alpha^{\prime \prime} \eta+\beta^{\prime \prime}\right. \\
& \left.\left.+\left(\alpha^{\prime}\right)^{2} \beta^{\prime}-\alpha^{\prime \prime} \rho_{\eta}+3 \alpha^{\prime} \alpha^{\prime \prime} \beta\right)-\frac{4}{9}\left(\alpha^{\prime} \gamma_{2}^{\prime} \eta+\beta^{\prime} \gamma_{2}^{\prime}\right)\right] \zeta+\left[\frac{2 \alpha^{\prime} \alpha^{\prime \prime}}{3}-\frac{8\left(\alpha^{\prime}\right)^{2} \gamma_{2}}{9}\right] \zeta^{2} .
\end{aligned}
$$

By (3.67) and (3.68), we can find $f$. 
Corollary 3.2. In terms of the notions in (3.12), we have the following solution of the equation (1.2) which blows up on the hypersurface $\sqrt{3}(\cos \alpha y+\sin \alpha z)+\beta=0$ :

$$
\begin{aligned}
& u=\zeta^{-2} x^{3}+\left[\rho \zeta^{-2}+\left(\alpha^{\prime} \eta+\beta^{\prime}\right) \zeta^{-1}+\frac{\gamma_{2}}{6}\right] x^{2}+\left[\frac{\rho^{2}}{3} \zeta^{-2}+\frac{\rho_{\eta}^{2}}{3}-\frac{2}{3}\left(\rho_{t}+\alpha^{\prime} \beta \rho_{\eta}\right)\right. \\
& \left.+\frac{2}{9} \gamma_{2} \rho-\frac{2}{3}\left[\alpha^{\prime \prime} \eta+\beta^{\prime \prime}+\left(\alpha^{\prime}\right)^{2} \beta-\alpha^{\prime} \rho_{\eta}\right] \zeta-\frac{4}{9}\left(\alpha^{\prime} \eta+\beta^{\prime}\right) \gamma_{2} \zeta\right] x \\
& +\frac{\rho^{3}}{27} \zeta^{-2}-\frac{\left(\alpha^{\prime} \eta+\beta^{\prime}\right) \rho^{2}}{9} \zeta^{-1}+\frac{\gamma_{2} \rho^{2}}{27} \ln \zeta-\frac{\gamma_{2} \rho_{\eta}^{2}+2 \gamma_{2}^{2} \rho}{54} \zeta^{2}(2 \ln \zeta-3) \\
& +\frac{\gamma_{2}^{3}}{324} \zeta^{4}(12 \ln \zeta-25)+\left[\frac{4}{9} \alpha^{\prime} \rho \rho_{\eta}+\frac{2\left(\alpha^{\prime} \eta+\beta^{\prime}\right)}{27}\left(9 \rho_{\eta}^{2}-6 \rho_{t}-6 \alpha^{\prime} \beta \rho_{\eta}+4 \gamma_{2} \rho\right)\right. \\
& \left.-\frac{4}{9} \rho\left(\alpha^{\prime \prime} \eta+\beta^{\prime \prime}+\left(\alpha^{\prime}\right)^{2} \beta\right)\right] \zeta(\ln \zeta-1)-\frac{2}{243}\left[29 \alpha^{\prime} \gamma_{2} \rho_{\eta}+\left(\alpha^{\prime} \eta+\beta^{\prime}\right)\left(20 \gamma_{2}^{2}-3 \gamma_{2}^{\prime}\right)\right. \\
& \left.-9\left(\alpha^{\prime}\right)^{2} \beta \gamma_{2}-3\left(\alpha^{\prime} \rho_{t \eta}+\gamma_{2}\left(\alpha^{\prime \prime} \eta+\beta^{\prime \prime}\right)+\alpha^{\prime \prime} \rho_{\eta}\right)\right] \zeta^{3}(6 \ln \zeta-11)+\left[\frac{2 \rho_{\eta} \rho_{t \eta}}{9}+\frac{2 \gamma_{2}^{\prime} \rho}{27}\right. \\
& -\frac{2}{9}\left(\rho_{t t}+2 \alpha^{\prime} \beta \rho_{t \eta}+\left(\alpha^{\prime} \beta\right)^{\prime} \rho_{\eta}+2\left(\alpha^{\prime} \beta\right)^{2} \gamma_{2}\right)+\frac{\gamma_{2} \rho_{t}}{27}+\frac{13 \alpha^{\prime} \beta \gamma_{2} \rho_{\eta}}{27}+\frac{\gamma_{2} \rho_{\eta}^{2}}{54} \\
& \left.+\frac{\gamma_{2}^{2} \rho}{81}-\frac{4}{9}\left(\alpha^{\prime} \eta+\beta^{\prime}\right)\left(\alpha^{\prime \prime} \eta+\beta^{\prime \prime}+\left(\alpha^{\prime}\right)^{2} \beta-\alpha^{\prime} \rho_{\eta}\right)-\frac{8}{27} \gamma_{2}\left(\alpha^{\prime} \eta+\beta^{\prime}\right)^{2}\right] \zeta^{2} \\
& +\left[\frac{2 \alpha^{\prime} \rho_{t \eta}}{27}+\frac{\gamma_{2}}{81}\left(19\left(\alpha^{\prime}\right)^{2} \beta-5 \alpha^{\prime \prime} \eta-5 \beta^{\prime \prime}\right)-\frac{13 \alpha^{\prime} \gamma_{2} \rho_{\eta}}{81}-\frac{2}{243} \gamma_{2}^{2}\left(\alpha^{\prime} \eta+\beta^{\prime}\right)\right. \\
& \left.-\frac{2}{27}\left(\alpha^{\prime \prime \prime} \eta+\beta^{\prime \prime \prime}+\left(\alpha^{\prime}\right)^{2} \beta^{\prime}-\alpha^{\prime \prime} \rho_{\eta}+3 \alpha^{\prime} \alpha^{\prime \prime} \beta\right)-\frac{4}{81}\left(\alpha^{\prime} \gamma_{2}^{\prime} \eta+\beta^{\prime} \gamma_{2}^{\prime}\right)\right] \zeta^{3} \\
& -\left[\frac{\gamma_{2} \gamma_{2}^{\prime}}{6}-\frac{\gamma_{2}^{\prime \prime}}{27}+\frac{7 \gamma_{2}^{3}}{486}-\frac{\alpha^{\prime} \alpha^{\prime \prime}}{9}+\frac{4\left(\alpha^{\prime}\right)^{2} \gamma_{2}}{27}\right] \zeta^{4},
\end{aligned}
$$

where $\rho$ is a third-order differentiable function as in (3.43), $\alpha$ is given in (3.45) and $\beta$ is any third-order differentiable function of $t$.

Finally, $\xi=0$ is the only solution of the equation (3.6) polynomial in $y$ and $z$. Under this assumption, the equations (3.7) and (3.8) become

$$
h_{y y}+h_{z z}=0, \quad g_{y y}+g_{z z}=4 h_{t}+4 h^{2} .
$$

The first equation is a Laplace equation whose solutions are called harmonic functions. It can be proved as (3.25) by power series that the general solution of the first equation is

$$
h=(\sigma+\sqrt{-1} \rho)(t, y+\sqrt{-1} z)+(\sigma-\sqrt{-1} \rho)(t, y-\sqrt{-1} z),
$$

where $\sigma(t, \mu)$ and $\rho(t, \mu)$ are complex functions in real variable $t$ and bar-homomorphic in complex variable $\mu$ (cf. (3.72)). Set

$$
w=y+\sqrt{-1} z, \quad \bar{w}=y-\sqrt{-1} z .
$$

Then the Laplace operator

$$
\partial_{y}^{2}+\partial_{z}^{2}=4 \partial_{w} \partial_{\bar{w}}
$$


The second equation in (3.78) is equivalent to

$$
\begin{aligned}
& \partial_{w} \partial_{\bar{w}}(g)=h_{t}+h^{2}=\left(\sigma_{t}+\sqrt{-1} \rho_{t}\right)(t, w)+\left(\sigma_{t}-\sqrt{-1} \rho_{t}\right)(t, \bar{w}) \\
& +[(\sigma+\sqrt{-1} \rho)(t, w)+(\sigma-\sqrt{-1} \rho)(t, \bar{w})]^{2} .
\end{aligned}
$$

Hence the general solution of the second equation in (3.78) is

$$
\begin{aligned}
& g=\int_{\overline{w_{1}}}^{\bar{w}} \int_{w_{1}}^{w}\left\{\left(\sigma_{t}+\sqrt{-1} \rho_{t}\right)\left(t, \mu_{1}\right)+\left(\sigma_{t}-\sqrt{-1} \rho_{t}\right)\left(t, \overline{\mu_{1}}\right)\right. \\
& \left.+\left[(\sigma+\sqrt{-1} \rho)\left(t, \mu_{1}\right)+(\sigma-\sqrt{-1} \rho)\left(t, \overline{\mu_{1}}\right)\right]^{2}\right\} d \mu_{1} d \overline{\mu_{1}} \\
& +(\kappa+\sqrt{-1} \omega)(t, w)+(\kappa-\sqrt{-1} \omega)(t, \bar{w}),
\end{aligned}
$$

where $\kappa(t, \mu)$ and $\omega(t, \mu)$ are complex functions in real variable $t$ and bar-homomorphic in complex variable $\mu$ (cf. (3.72)) and $w_{1}$ is a complex constant. Furthermore, (3.9) becomes

$$
\partial_{w} \partial_{\bar{w}}(f)=\frac{1}{2}\left(g_{t}+g h\right)
$$

Observe that

$$
\begin{aligned}
& g_{t}=\int_{\overline{w_{1}}}^{\bar{w}} \int_{w_{1}}^{w}\left[2 ( ( \sigma + \sqrt { - 1 } \rho ) ( t , \mu _ { 1 } ) + ( \sigma - \sqrt { - 1 } \rho ) ( t , \overline { \mu _ { 1 } } ) ) \left(\left(\sigma_{t}+\sqrt{-1} \rho_{t}\right)\left(t, \mu_{1}\right)\right.\right. \\
& \left.\left.+\left(\sigma_{t}-\sqrt{-1} \rho_{t}\right)\left(t, \overline{\mu_{1}}\right)\right)+\left(\sigma_{t t}+\sqrt{-1} \rho_{t t}\right)\left(t, \mu_{1}\right)+\left(\sigma_{t t}-\sqrt{-1} \rho_{t t}\right)\left(t, \overline{\mu_{1}}\right)\right] d \mu_{1} d \overline{\mu_{1}} \\
& +\left(\kappa_{t}+\sqrt{-1} \omega_{t}\right)(t, w)+\left(\kappa_{t}-\sqrt{-1} \omega_{t}\right)(t, \bar{w}) .
\end{aligned}
$$

Thus the general solution of the equation (3.9) is

$$
\begin{aligned}
& f=\frac{1}{2} \int_{\overline{w_{2}}}^{\bar{w}} \int_{w_{2}}^{w}\left\{\int _ { \overline { w _ { 1 } } } ^ { \overline { \mu _ { 2 } } } \int _ { w _ { 1 } } ^ { \mu _ { 2 } } \left[2\left((\sigma+\sqrt{-1} \rho)\left(t, \mu_{1}\right)+(\sigma-\sqrt{-1} \rho)\left(t, \overline{\mu_{1}}\right)\right)\right.\right. \\
& \times\left(\left(\sigma_{t}+\sqrt{-1} \rho_{t}\right)\left(t, \mu_{1}\right)+\left(\sigma_{t}-\sqrt{-1} \rho_{t}\right)\left(t, \overline{\mu_{1}}\right)\right)+\left(\sigma_{t t}+\sqrt{-1} \rho_{t t}\right)\left(t, \mu_{1}\right) \\
& \left.+\left(\sigma_{t t}-\sqrt{-1} \rho_{t t}\right)\left(t, \overline{\mu_{1}}\right)\right] d \mu_{1} d \overline{\mu_{1}}+\left(\kappa_{t}+\sqrt{-1} \omega_{t}\right)\left(t, \mu_{2}\right)+\left(\kappa_{t}-\sqrt{-1} \omega_{t}\right)\left(t, \overline{\mu_{2}}\right) \\
& +\left[(\sigma+\sqrt{-1} \rho)\left(t, \mu_{2}\right)+(\sigma-\sqrt{-1} \rho)\left(t, \overline{\mu_{2}}\right)\right]\left[(\kappa+\sqrt{-1} \omega)\left(t, \mu_{2}\right)\right. \\
& +(\kappa-\sqrt{-1} \omega)\left(t, \overline{\mu_{2}}\right)+\int_{\overline{w_{1}}}^{\overline{\mu_{2}}} \int_{w_{1}}^{\mu_{2}}\left\{\left(\sigma_{t}+\sqrt{-1} \rho_{t}\right)\left(t, \mu_{1}\right)+\left(\sigma_{t}-\sqrt{-1} \rho_{t}\right)\left(t, \overline{\mu_{1}}\right)\right. \\
& \left.\left.\left.+\left[(\sigma+\sqrt{-1} \rho)\left(t, \mu_{1}\right)+(\sigma-\sqrt{-1} \rho)\left(t, \overline{\mu_{1}}\right)\right]^{2}\right\} d \mu_{1} d \overline{\mu_{1}}\right]\right\} d \mu_{2} d_{\overline{\mu_{2}}} \\
& +(\chi+\sqrt{-1} \nu)(t, w)+(\chi-\sqrt{-1} \nu)(t, \bar{w})
\end{aligned}
$$

where $\chi(t, \mu)$ and $\nu(t, \mu)$ are complex functions in real variable $t$ and bar-homomorphic in complex variable $\mu$ and $w_{2}$ is a complex constant. 
THEOREM 3.3. In terms of the notions in (3.79), the following is a solution polynomial in $x$ of the equation (1.2):

$$
\begin{aligned}
& u=[(\sigma+\sqrt{-1} \rho)(t, w)+(\sigma-\sqrt{-1} \rho)(t, \bar{w})] x^{2}+\left\{\int _ { \overline { w _ { 1 } } } ^ { \overline { w } } \int _ { w _ { 1 } } ^ { w } \left\{\left(\sigma_{t}+\sqrt{-1} \rho\right)\left(t, \mu_{1}\right)\right.\right. \\
& \left.+\left(\sigma_{t}-\sqrt{-1} \rho\right)\left(t, \overline{\mu_{1}}\right)+\left[(\sigma+\sqrt{-1} \rho)\left(t, \mu_{1}\right)+(\sigma-\sqrt{-1} \rho)\left(t, \overline{\mu_{1}}\right)\right]^{2}\right\} d \mu_{1} d \overline{\mu_{1}} \\
& +(\kappa+\sqrt{-1} \omega)(t, w)+(\kappa-\sqrt{-1} \omega)(t, \bar{w})\} x+\frac{1}{2} \int_{\overline{w_{2}}}^{\bar{w}} \int_{w_{2}}^{w}\left\{\int_{\overline{w_{1}}}^{\overline{\mu_{2}}} \int_{w_{1}}^{\mu_{2}}\right. \\
& {\left[2 ( ( \sigma + \sqrt { - 1 } \rho ) ( t , \mu _ { 1 } ) + ( \sigma - \sqrt { - 1 } \rho ) ( t , \overline { \mu _ { 1 } } ) ) \left(\left(\sigma_{t}+\sqrt{-1} \rho_{t}\right)\left(t, \mu_{1}\right)\right.\right.} \\
& \left.\left.+\left(\sigma_{t}-\sqrt{-1} \rho_{t}\right)\left(t, \overline{\mu_{1}}\right)\right)+\left(\sigma_{t t}+\sqrt{-1} \rho_{t t}\right)\left(t, \mu_{1}\right)+\left(\sigma_{t t}-\sqrt{-1} \rho_{t t}\right)\left(t, \overline{\mu_{1}}\right)\right] d \mu_{1} d \overline{\mu_{1}} \\
& +\left(\kappa_{t}+\sqrt{-1} \omega_{t}\right)\left(t, \mu_{2}\right)+\left(\kappa_{t}-\sqrt{-1} \omega_{t}\right)\left(t, \overline{\mu_{2}}\right)+\left[(\sigma+\sqrt{-1} \rho)\left(t, \mu_{2}\right)\right. \\
& \left.+(\sigma-\sqrt{-1} \rho)\left(t, \overline{\mu_{2}}\right)\right]\left[(\kappa+\sqrt{-1} \omega)\left(t, \mu_{2}\right)+(\kappa-\sqrt{-1} \omega)\left(t, \overline{\mu_{2}}\right)\right. \\
& +\int_{\overline{w_{1}}}^{\overline{\mu_{2}}} \int_{w_{1}}^{\mu_{2}}\left\{\left(\sigma_{t}+\sqrt{-1} \rho_{t}\right)\left(t, \mu_{1}\right)+\left(\sigma_{t}-\sqrt{-1} \rho_{t}\right)\left(t, \overline{\mu_{1}}\right)\right. \\
& \left.\left.\left.+\left[(\sigma+\sqrt{-1} \rho)\left(t, \mu_{1}\right)+(\sigma-\sqrt{-1} \rho)\left(t, \overline{\mu_{1}}\right)\right]^{2}\right\} d \mu_{1} d \overline{\mu_{1}}\right]\right\} d \mu_{2} d_{\overline{\mu_{2}}} \\
& +(\chi+\sqrt{-1} \nu)(t, w)+(\chi-\sqrt{-1} \nu)(t, \bar{w}),
\end{aligned}
$$

where $\sigma(t, \mu), \rho(t, \mu), \kappa(t, \mu), \omega(t, \mu), \chi(t, \mu)$ and $\nu(t, \mu)$ are complex functions in real variable $t$ and bar-homomorphic in complex variable $\mu$ (cf. (3.72)). Moreover, the above solution is smooth (analytic) if all $\sigma, \rho, \kappa, \omega, \chi$ and $\nu$ are smooth (analytic) functions. In particular, any solution of the equation (1.2) polynomial in $x, y, z$ must be of the form (3.87) in which $\sigma, \rho, \kappa, \omega, \chi$ and $\nu$ are polynomial in $\mu$.

REMARK 3.4. In addition to the solutions in Theorems 3.1 and 3.2, the equation (1.2) has the following simple solution:

$$
u=\wp_{\iota}(\sqrt{3}(a y+b z)) x^{3},
$$

where $\wp_{\iota}(w)$ is the Weierstrass's elliptic function satisfying (2.39) and $a, b$ are real constants such that $a^{2}+b^{2}=1$.

\section{REFERENCES}

[I] N. H. Ibragimov, Lie Group Analysis of Differential Equations, Volume 1, CRC Handbook, CRC Press, 1995.

[LRT] C. C. Lin, E. Reissner and H. S. Tsien, On two-dimensional non-steady motion of a slender body in a compressible fluid, J. Math, Phys. 27 (1948), no. 3, 220. MR0026499 (10:162e)

[Kp1] P. Kucharczyk, Group properties of the "short waves" equations in gas dynamics, Bull. Acad. Polon. Sci., Ser. Sci. Techn. XIII (1965), no. 5, 469.

[Kp2] P. Kucharczyk, Teoria Grup Liego w Zastosowaniu do Rówman Rózniczkowych Czaskowych, IPPT Polish Academy of Sciences, Warsaw, 65, 1967.

[M1] E. V. Mamontov, On the theory of nonstationary transonic flows, Dokl. Acad. Nauk SSSR 185 (1969), no. 3, 538 .

[M2] E. V. Mamontov, Analytic perturbations in a nonstationary transonic stream, Dinamika Splosn. Sredy Vyp. 10 (1972), 217-222. MR0459234 (56:17428)

[RS] O. S. Ryzhov and G. M. Shefter, On unsteady gas flows in Laval nozzles, Soviet Physics. Dokl. 4 (1959), 939-942. MR0119694 (22:10455) 
[Sg] G. D. Sevost'janov, An equation for nonstationary transonic flows of an ideal gas, Izv. Acad. Nauk SSSR Meh. Zidk. Gaza, 1977, no.1, 105-109. MR0475254 (57:14871)

[Sv] S. V. Sukhinin, Group property and conservation laws of the equation of transonic motion of gas, Dinamika Splosh. Sredi 36 (1978), 130. MR539014 (80h:76037) 